\title{
SISTEMA URBANO ARGENTINO
}

\section{Prof.: Aníbal Marcelo Mignone Prof.: Alejandra Helena Torre Geraldi \\ Cátedra: Geografía Argentina Profesorado y Licenciatura en Geografía UNNE}


- Material de consulta de cátedra para los alumnos de las carreras del Profesorado y de la Licenciatura en Geografía de la UNNE.

- Esta presentación, es una elaboración propia, generada a partir de la compilación de bibliografía impresa y de páginas web. 


\section{SISTEMA URBANO}

- Sistema interdependiente de ciudades.

- Las ciudades son consideradas como focos de producción, distribución, consumo y organización del sistema (Bouchert, Bourne y Sinclair, 1986 citado por Meichtry 1998).

Fuente: Meichtry, 1998. 


\section{Urbanización}

- Fenómeno relativamente moderno en la historia de la sociedad.

- Significa la existencia de ciudades como unidades localizadas que presentan una alta concentración de personas con modos de vida primariamente no agrarios.

- Proceso de cambio desde una estructura celular, desconectada, hacia un sistema integrado e interdependiente cual el modo urbano penetra "cada esfera de la vida personal y colectiva" (Hawley, 1971 citado por Meichtry, 1998).

- Proceso que concentra a la población y las actividades en las ciudades, lo que conlleva a cambios demográficos, económicos y culturales.

Fuente: Meichtry, 1998. 


\section{Urbanización}

El proceso de urbanización, con las características que reviste actualmente, se inició a mediados del siglo XIX y alcanzó su máximo desarrollo en el siglo $\mathrm{XX}$.

Fuente: Meichtry, 1998.

\section{Población residente en ciudades de más de 5000 habitantes}

- 1850

- 1900

- 1950

- Actualidad

$$
6,4 \%
$$

$13,6 \%$

$29,8 \%$

$50 \%$
Fuente: ONU 1978, Banco Mundial 1999. 


\section{Urbanización}

\section{Proceso de concentración de población por:}

- Multiplicación de puntos de concentración

- Aumento de las concentraciones individuales

Fuente: Meichtry, 1998. 
- Las ciudades se relacionan y articulan entre sí de acuerdo con el volumen de población que poseen (tamaño), las actividades que desarrollan (funciones) y las distancias a las cuales se encuentran unas de otras.

- Se organizan y comandan un territorio, para lo cuál se establece necesariamente un ordenamiento jerárquico. 


\section{Ventajas}

- A medida que aumenta el tamaño de la ciudad ( $n^{\circ}$ de habitantes):

- Se produce una diversificación de las actividades económicas.

- Se incrementa la demanda de servicios e inversiones en infraestructura de transporte.

\section{Desventajas}

- Aumento de las desigualdades sociales.

- Incremento de la contaminación.

- Dificultad para acceder al empleo. 


\section{ETAPAS DEL SISTEMA URBANO ARGENTINO}




\section{Período Hispánico (fines del siglo XVI- mediados siglo XVIII)}

- Las corrientes colonizadoras

- Las ciudades principales datan de la época de la colonización.

Respondieron a

- Necesidad de apoyo de los establecimientos mineros de Potosí.

- Control del bajo Paraná, en la frontera con las posesiones portuguesas.

- Anhelo de disponer de una salida al océano y de comunicación con España.

Fuente: Vapñarsky y Gorojovsky 1990; Bortagaray, 1992; Erbiti, 1997. 


\section{Período Hispánico (fines del siglo XVI- mediados siglo XVIII)}

Los núcleos urbanos:

- Configuraron una malla de trama abierta.

- Ocupación discontínua del espacio.

- Áreas de influencia poco extensas.

Regiones más importantes:

- Noroeste

- Cuyo

- Córdoba

Fuente: Vapñarsky y Gorojovsky 1990; Bortagaray, 1992; Erbiti, 1997.

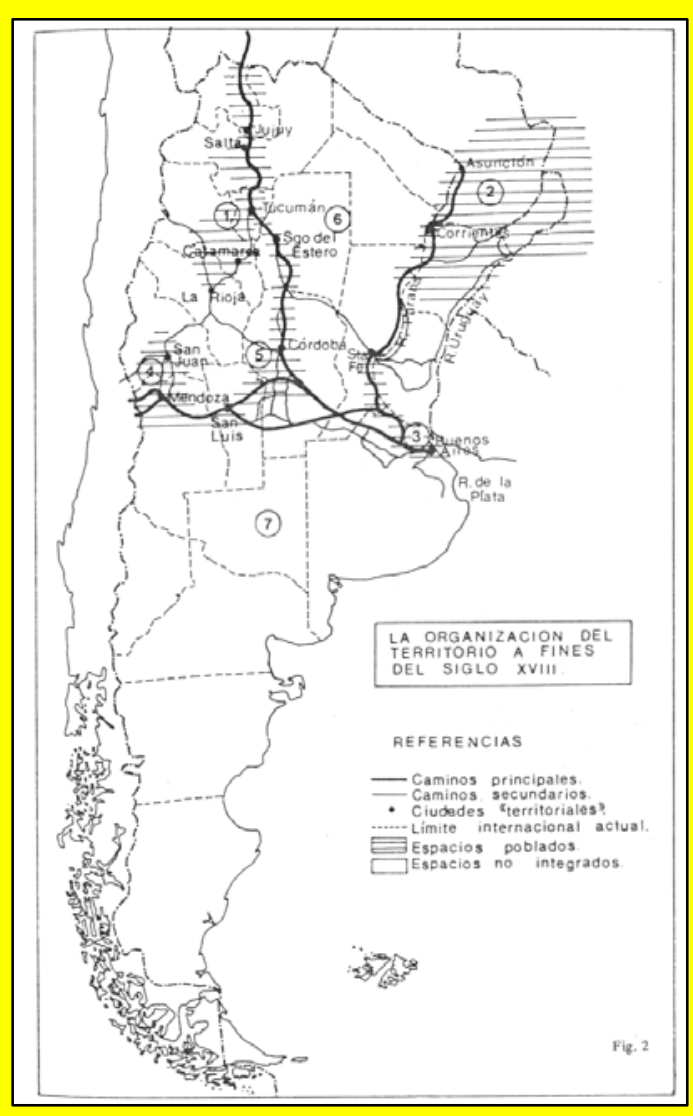

Fuente: Bortagaray, 1992:155.

Publicado en formato digital: Prof. Aníbal Marcelo Mignone y Prof. Alejandra H. Torre Geraldi. SISTEMA URBANO ARGENTINO. Producción en Docencia. Revista Geográfica Digital. IGUNNE. Facultad de Humanidades. UNNE. Año 11. No 22. Julio - Diciembre 2014. Resistencia, Chaco. 


\section{Período Independentista (mediados del siglo XVIII hasta 1880)}

\section{Virreinato del Río de la Plata}

\section{Puerto al tráfico de ultramar}

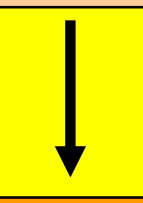

\section{Buenos Aires}

Fuente: Vapñarsky y Gorojovsky 1990; Bortagaray, 1992; Erbiti, 1997.

Publicado en formato digital: Prof. Aníbal Marcelo Mignone y Prof. Alejandra H. Torre Geraldi. SISTEMA URBANO ARGENTINO. Producción en Docencia. Revista Geográfica Digital. IGUNNE. Facultad de Humanidades. UNNE. Año 11. № 22. Julio - Diciembre 2014. Resistencia, Chaco.

En: http://hum.unne.edu.ar/revistas/geoweb/default.htm 


\section{Período Independentista (mediados del siglo XVIII hasta 1880)}

- Quiebre del eje Potosí- Buenos Aires

- Importación de productos manufacturados

- Ruptura del equilibrio regional

- Buenos Aires se convierte en capital del país (1880) y manejo a su arbitrio el comercio exterior. 


\section{Período Independentista (mediados del siglo XVIII hasta 1880)}

"Al controlar el puerto controlaba el desarrollo industrial, única opción abierta a las ciudades del interior. $Y$ al controlar la aduana se apoderaba prácticamente de la totalidad de las rentas nacionales. Cerraba así cualquier camino a la inversión estatal en provecho del Interior. He aquí el verdadero origen de la macrocefalía porteña" (Vapñarsky y Gorojovsky, 1990, citado por Erbiti, 1997)" 


\section{Período de la Factoría Agroexportadora (1880-1950)}

- El desarrollo de los ferrocarriles

- Inmigración europea fundamentalmente a Buenos Aires y la región pampeana.

- 1869- 1947 mayor crecimiento de las ciudades pampeanas

- Migraciones internas hacia los principales centros industriales del país.

Fuente: Vapñarsky y Gorojovsky 1990; Bortagaray, 1992; Erbiti, 1997.

Publicado en formato digital: Prof. Aníbal Marcelo Mignone y Prof. Alejandra H. Torre Geraldi. SISTEMA URBANO ARGENTINO. Producción en Docencia. Revista Geográfica Digital. IGUNNE. Facultad de Humanidades. UNNE. Año 11. No 22. Julio - Diciembre 2014. Resistencia, Chaco. En: http://hum.unne.edu.ar/revistas/geoweb/default.htm 


\section{Los cambios desde 1950}

- A partir de 1950 el sistema de asentamiento sufrió una profunda transformación:

- El Gran Buenos Aires sigue siendo diez veces más populosa que cualquier otra aglomeración.

- Disminuyó la macrocefalía.

- La población en ciudades de $\mathbf{5 0 . 0 0 0 ~ o ~ m a s ~ h a b i t a n t e s ~}$ (aglomeraciones de tamaño intermedio, o ATIs) se triplicó.

- Fenómeno de Contraurbanización.

Fuente: Vapñarsky y Gorojovsky 1990; Bortagaray, 1992; Erbiti, 1997. 


\section{Los cambios desde 1950}

- Se desarrollan las carreteras y el mercado automotor.

- Se cierran ramales de los ferrocarriles y se clausuran estaciones.

- La demanda de empleo industrial.

- Localización de la población rural en ciudades próximas a su lugar de trabajo. 


\section{Consecuencias}

- Ha disminuido la distancia promedio entre la residencia de cada habitante del país.

- Crecimiento de algunos centros de servicios puntuales especializados.

- Mercados de trabajo marcadamente diversificado.

- Se ha multiplicado el numero de centros de distribución de bienes de consumo masivo hasta el punto de servir a casi toda la población nacional. 


\section{Clasificación de las Aglomeraciones por Tamaño}

\begin{tabular}{|l|l|l|l|}
\hline \multirow{2}{*}{$\begin{array}{l}\text { Población } \\
\text { rural }\end{array}$} & Categorías & Subcategorías & Población (hab) \\
\hline & & $\begin{array}{l}\text { Población } \\
\text { dispersa }\end{array}$ & $\begin{array}{l}\text { Dispersa } \\
\text { en campo abierto }\end{array}$ \\
\cline { 3 - 5 } & \multirow{2}{*}{$\begin{array}{l}\text { Pueblos } \\
\text { pequeños }\end{array}$} & Hasta 1999 \\
\hline \multirow{3}{*}{$\begin{array}{l}\text { Población } \\
\text { urbana }\end{array}$} & \multirow{2}{*}{$\begin{array}{l}\text { II - Aglomeraciones de } \\
\text { tamaño intermedio (ATIs) }\end{array}$} & $\begin{array}{l}\text { Pueblos } \\
\text { grandes }\end{array}$ & 2000 a 19.999 \\
\cline { 3 - 5 } & \multirow{2}{*}{\begin{tabular}{l} 
ITIS mayores \\
\cline { 3 - 5 }
\end{tabular}} & $\begin{array}{l}\text { Ciudades } \\
\text { pequeñas }\end{array}$ & 20.000 a 49.999 \\
\cline { 3 - 5 } & \multirow{2}{*}{$\begin{array}{l}\text { III - Aglomeraciones de } \\
\text { más de 1 millón de hab. }\end{array}$} & & 50.000 a 499.999 \\
\hline
\end{tabular}

Fuente: Vapñarsky y Gorojosky, 1990 adaptado por Erbiti, 2007. 


\section{Distribución de la población argentina según categoría de ciudades. (En miles de habitantes y en porcentaje respecto del total).}

\begin{tabular}{|c|c|c|c|c|c|c|}
\hline & 1947 & 1960 & 1970 & 1980 & 1991 & 2001 \\
\hline I GBA & $\begin{array}{l}5150 \\
(30 \%)\end{array}$ & $\begin{array}{l}6750 \\
(34 \%)\end{array}$ & $\begin{array}{l}8450 \\
(36 \%)\end{array}$ & $\begin{array}{l}9950 \\
(36 \%)\end{array}$ & $\begin{array}{l}10935 \\
(34 \%)\end{array}$ & $\begin{array}{l}11783 \\
(32 \%)\end{array}$ \\
\hline $\begin{array}{l}\text { II Pobl. en ATIs } \\
\text { (excepto GBA) }\end{array}$ & $\begin{array}{l}2700 \\
(16 \%)\end{array}$ & $\begin{array}{l}4100 \\
(20 \%)\end{array}$ & $\begin{array}{l}5800 \\
(25 \%)\end{array}$ & $\begin{array}{l}8000 \\
(28 \%)\end{array}$ & $\begin{array}{l}11001 \\
(34 \%)\end{array}$ & $\begin{array}{l}13424 \\
(36 \%)\end{array}$ \\
\hline $\begin{array}{l}\text { III Pobl. Dispersa, aglom. } \\
\text { menores a } 50.000 \text { hab. }\end{array}$ & $\begin{array}{l}9000 \\
(53 \%)\end{array}$ & $\begin{array}{l}9150 \\
(46 \%)\end{array}$ & $\begin{array}{l}9100 \\
(39 \%)\end{array}$ & $\begin{array}{l}10000 \\
(36 \%)\end{array}$ & $\begin{array}{l}10679 \\
(33 \%)\end{array}$ & $\begin{array}{l}12075 \\
(32 \%)\end{array}$ \\
\hline Número de ATIs & 15 & 23 & 31 & 41 & 54 & 63 \\
\hline
\end{tabular}




\section{Población urbana en Argentina}

- Tendencia a la concentración de actividades en la región pampeana, mucho antes del comienzo de la etapa agro-exportadora.

- Se planteó un proceso de desigualdad en la distribución de la población y las actividades económicas.

- Sostenido proceso de urbanización, impulsado a partir del modelo agro-exportador e incrementado durante la sustitución de importaciones

\begin{tabular}{|cc|}
\hline $\begin{array}{c}\text { Población urbana } \\
\text { en Argentina }\end{array}$ \\
\hline AÑO & $\%$ \\
\hline 1869 & $\mathbf{2 8}$ \\
\hline 1895 & 37 \\
\hline 1914 & 53 \\
\hline 1947 & $\mathbf{6 2}$ \\
\hline 1960 & $\mathbf{7 2}$ \\
\hline 1970 & $\mathbf{7 9}$ \\
\hline 1980 & $\mathbf{8 3}$ \\
\hline 1991 & $\mathbf{8 8}$ \\
\hline 2001 & 90 \\
\hline 2010 & $\mathbf{9 1}$ \\
\hline
\end{tabular}

Fuente: Velázquez, 2007; INDEC, 2013. 


\section{Alta primacía}

- Concepto elaborado por Jefferson (1939)

- Ciudad Líder (Ciudad Primada)

- Mayor complejidad

- La ciudad mayor del sistema excede más del doble a la que le sigue en magnitud de población

- Índice de Primacía: Población de la primera ciudad

$$
\text { C. } 2+\mathrm{C} 3+\mathrm{C} 4
$$

Fuente: Meichtry, 1998. 


\section{Alta primacía}

- La primacía urbana entendida como la relación entre la ciudad mayor y el resto de la malla urbana es menos sensible que la concentración urbana a los cambios económicos.

- Tiene un comportamiento mucho más resistente al cambio, que la concentración urbana, entendida como la distribución general de la población por tamaños de la ciudad.

Fuente: Meichtry, 1998. 


\section{Relación de primacía de Buenos Aires en el sistema urbano Argentino}

- Desde antes de la etapa agroexportadora y hasta la sustitución de importaciones la configuración espacial Argentina promovió el incremento de la primacía urbana de Buenos Aires.

- Hacia fines de los setenta, el desmantelamiento de la trama productiva afectó en mayor medida a las grandes ciudades, neutralizando parcialmente este proceso secular.

\begin{tabular}{|ll|}
\hline 1869 & 6,2 a 1 (Córdoba) \\
1895 & 7,5 a 1 (Rosario) \\
1914 & 8,8 a 1 (Rosario) \\
1947 & 9,3 a 1 (Rosario) \\
1960 & 10,3 a 1 (Rosario) \\
1970 & 10,4 a 1 (Rosario) \\
1980 & 10,1 a 1 (Córdoba) \\
1991 & 10,2 a 1 (Córdoba) \\
2001 & 10,1 a 1 (Córdoba) \\
2010 & 8,8 a 1 (Córdoba) \\
\hline
\end{tabular}

Fuente: Velázquez, 2007. INDEC 2013 
- Esta situación de primacía trajo consigo la implementación de una serie de medidas de "desarrollo regional".

- En la década del `60: la creación del Consejo Nacional de Desarrollo (CONADE), que se proponía contribuir a un desarrollo regional más "equilibrado.

- En los `70 surge la propuesta de regímenes de promoción industrial en provincias extra-pampeanas. Su implementación se concreta una década más tarde en el Noroeste (Catamarca y La Rioja), Cuyo (San Juan y San Luis) y un régimen especial en un territorio nacional patagónico (Tierra del Fuego). 


\section{Regla rango-tamaño}

Todos los sistemas urbanos presentan una distribución jerárquica.

Singer (1936) identificó el ordenamiento y Zipf $(1941,1949)$ colocó el esquema bajo el supuesto de la regla rangotamaño.

"Los centros de un sistema urbano tienen siguen un particular ordenamiento de tamaños, en donde el número de habitantes de la localidad en la posición $n$ tiene $1 / n$ la población de la ciudad mayor"

Fuente: Meichtry, 1998. 


\section{Regla Rango-Tamaño}

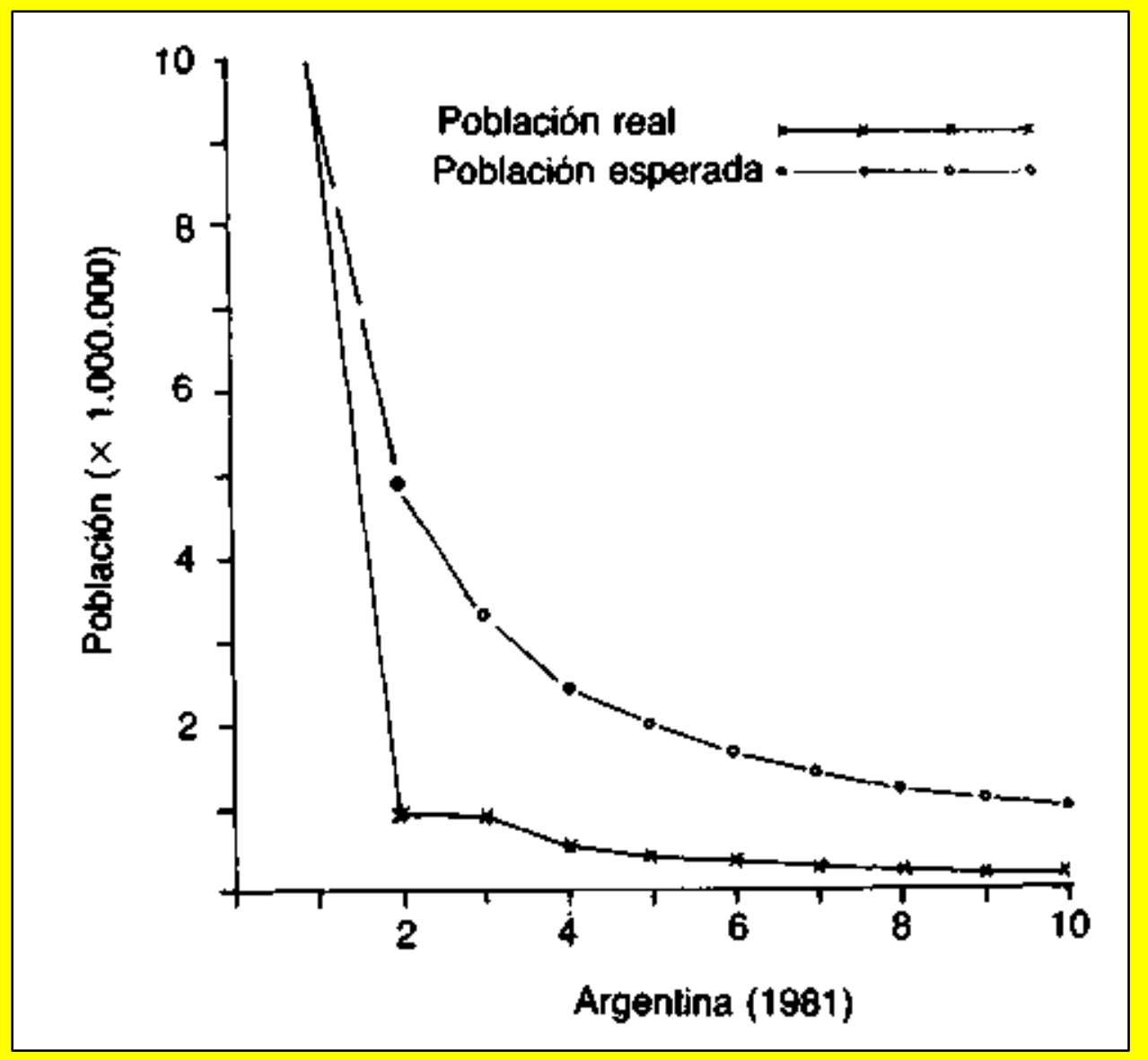

Fuente: Zárate Martín, 2000. 


\section{Macrocefalía}

- La población de la ciudad mayor excede a la suma de la población conjunta de los demás núcleo urbanos

- Se define como la existencia de ciudades mayores (o "cabezas urbanas") "desproporcionadamente" grandes con respecto al sistema de ciudades que le sirve de soporte".

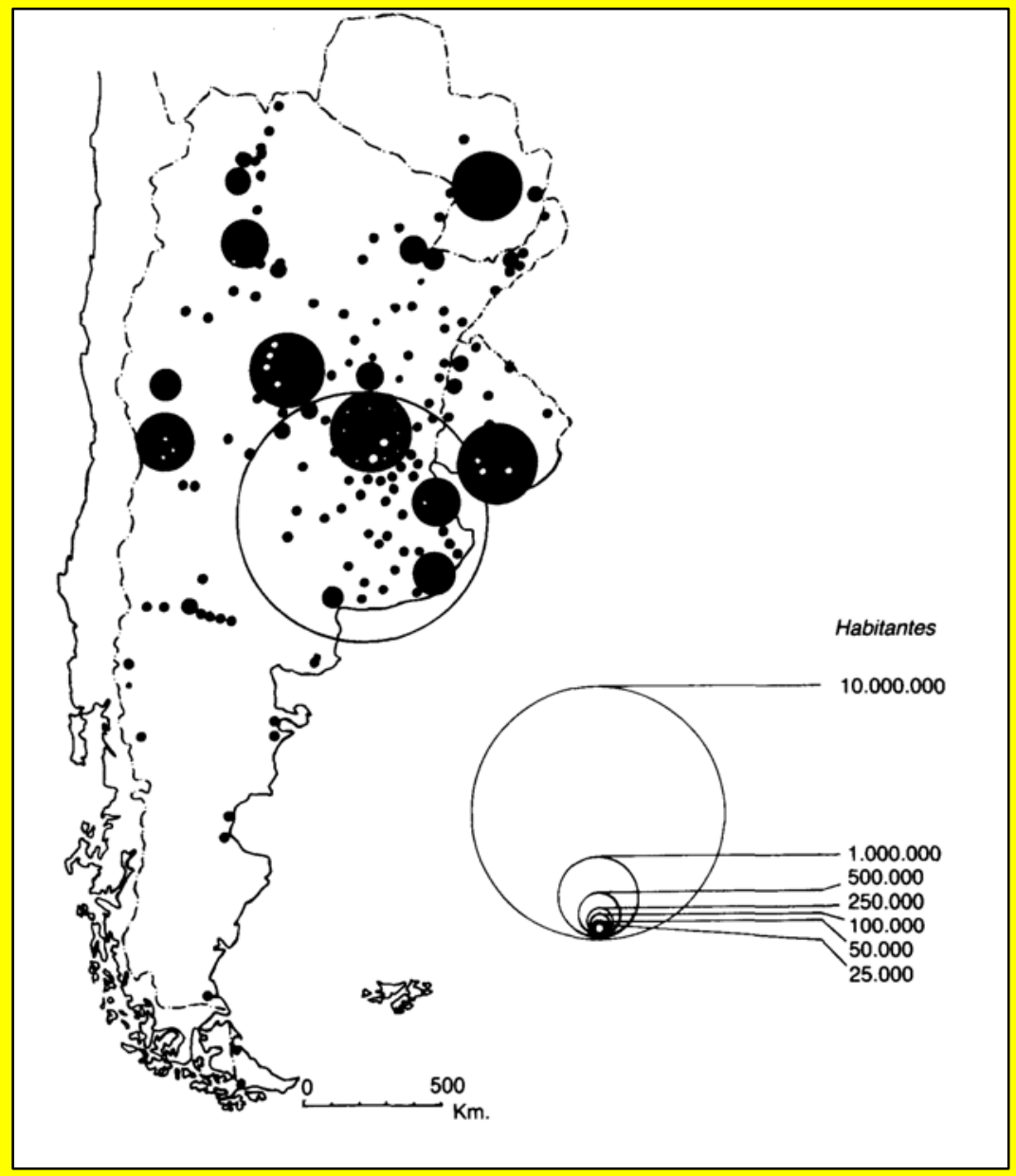

Fuente: Meichtry, 1998.

Fuente: Zárate Martín, 2000.

Publicado en formato digital: Prof. Aníbal Marcelo Mignone y Prof. Alejandra H. Torre Geraldi. SISTEMA URBANO ARGENTINO. Producción en Docencia. Revista Geográfica Digital. IGUNNE. Facultad de Humanidades. UNNE. Año 11. № 22. Julio - Diciembre 2014. Resistencia, Chaco. En: http://hum.unne.edu.ar/revistas/geoweb/default.htm 
- En las últimas décadas se está comprobando una fuerte tendencia de crecimiento en centros intermedios especialmente en regiones extra-pampeanas.

Las ATls protagonizaron la transformación por cuatro razones:

- El enorme incremento absoluto y relativo de su población conjunta.

- La multiplicación de su número.

- El crecimiento diferencial de ATIs antiguas (las que ya lo eran en 1950) y nuevas, mayor en las nuevas.

- La dispersión geográfica de las nuevas ATIs.

Fuente: Velázquez, 2007; Usach y Garrido, 2009.

Publicado en formato digital: Prof. Aníbal Marcelo Mignone y Prof. Alejandra H. Torre Geraldi. SISTEMA URBANO ARGENTINO. Producción en 


\section{Las ATIs}

\section{Factores que estimularon el crecimiento:}

- El componente espacial de la reciente reestructuración industrial.

- Las políticas de promoción industrial y regional.

- El crecimiento del empleo público y de actividades económicas nuevas en las capitales de provincia.

- El turismo.

- La creciente atracción de migrantes.

Fuente: Velázquez, 2007; Usach y Garrido, 2009.

Publicado en formato digital: Prof. Aníbal Marcelo Mignone y Prof. Alejandra H. Torre Geraldi. SISTEMA URBANO ARGENTINO. Producción en Docencia. Revista Geográfica Digital. IGUNNE. Facultad de Humanidades. UNNE. Año 11. № 22. Julio - Diciembre 2014. Resistencia, Chaco. En: http://hum.unne.edu.ar/revistas/geoweb/default.htm 


\section{Distribución regional de las ciudades intermedias} (ATIs)

\begin{tabular}{|c|c|c|c|c|c|c|}
\hline & NOA & NEA & PATAG. & CUYO & PAMPA & TOTAL \\
\hline $\mathbf{1 9 4 7}$ & 3 & 2 & - & 2 & 8 & 15 \\
\hline $\mathbf{2 0 0 1}$ & 9 & 8 & 9 & 5 & 32 & 63 \\
\hline
\end{tabular}

Fuente: Velázquez, 2007

Publicado en formato digital: Prof. Aníbal Marcelo Mignone y Prof. Alejandra H. Torre Geraldi. SISTEMA URBANO ARGENTINO. Producción en Docencia. Revista Geográfica Digital. IGUNNE. Facultad de Humanidades. UNNE. Año 11. № 22. Julio - Diciembre 2014.

En: http://hum.unne.edu.ar/revistas/geoweb/default.htm 


\section{Contraurbanización}

\section{Contraurbanización (geógrafos): proceso de redistribución de la población favoreciendo destinos no metropolitanos.}

Contraurbanización (Brian Berry): se entiende como cambio brusco en los modelos de poblamiento urbano en los países con un largo historial industrial que implica la salida de población de los centros metropolitanos más antiguos y más densamente poblados y el aumento paralelo de otras áreas metropolitanas.

Se da un proceso de redistribución de la población; se produce el crecimiento basado en ciudades medianas y pequeñas mucho más homogéneamente distribuidas en el territorio nacional.

Fuente: Meichtry, 1998. 


\section{Contraurbanización en Argentina}

- Disminuye la población relativa de Buenos Aires.

- Descentralización de los grandes centros urbanos hacia áreas no metropolitanas.

- Migración de retorno: movimientos hacia provincias periféricas con baja densidad.

- Las provincias comienzan a retener población.

- Problemas en las grandes ciudades: costos de vida, desempleo, inseguridad, contaminación, redes familiares, crecimiento social. 


\title{
Contraurbanización en Argentina
}

Ejemplos de políticas nacionales destinadas a disminuir paulatinamente la primacía de la $1^{\text {a }}$ ciudad:

- Planes de Concentración Industrial y de Promoción de Desarrollo Regional.

- Ley 18575/70 (Zonas y áreas de frontera).

\author{
Fuente: Meichtry, 1998.
}




\section{Organización funcional del sistema urbano}

\begin{abstract}
Morfología $\longrightarrow$ Concentrado
Jerarquía $\longrightarrow$ Altamente desequilibrado

Función $\longrightarrow$ Centralizado y radiocéntrico

Subsistemas $\longrightarrow$ No consolidados, con escasa interacción entre sí y gran dependencia del área central

Textura $\longrightarrow$ Diferencial

Precarias relaciones con los sistemas urbanos de los países limítrofes
\end{abstract}

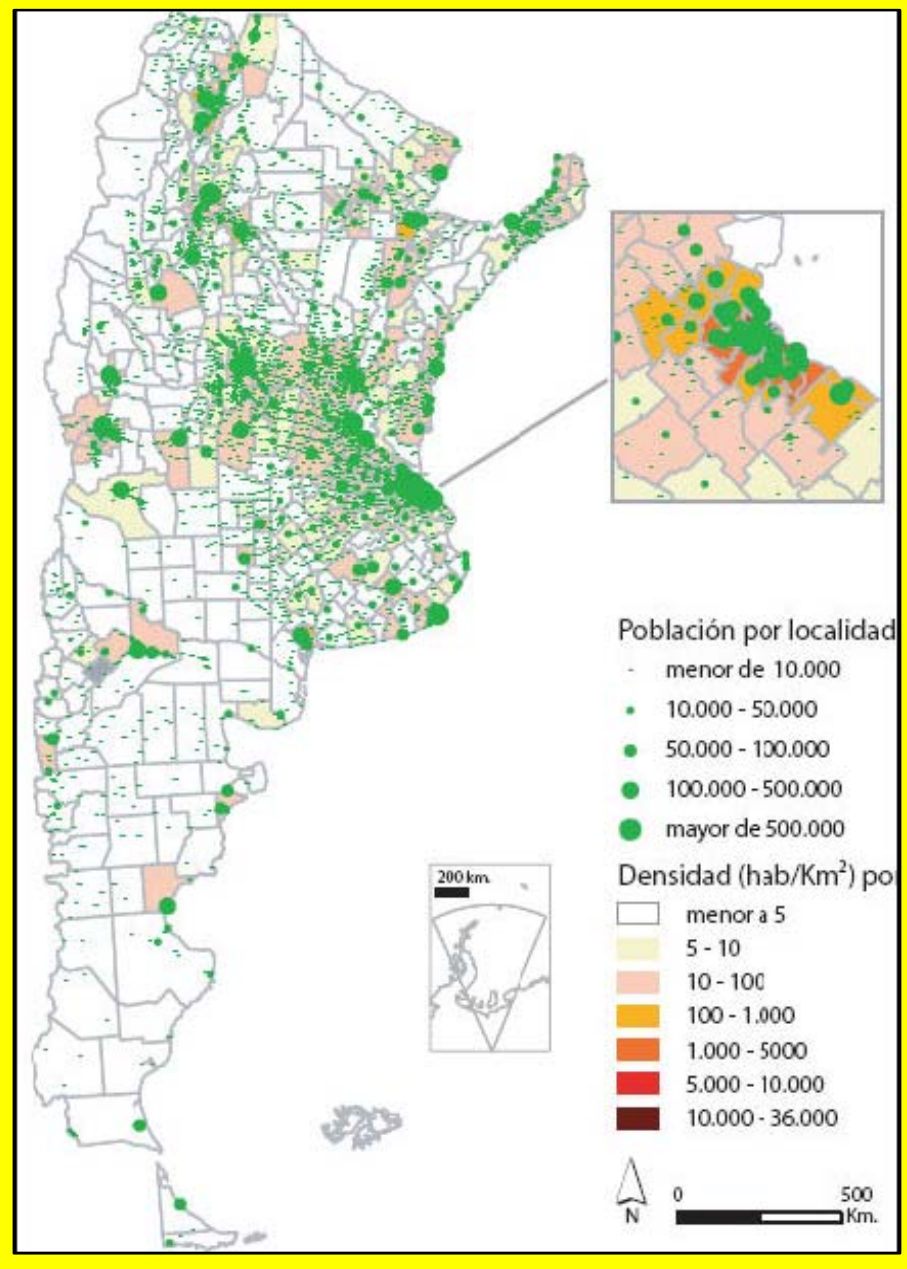

Fuente: Usach y Garrido, 2009

Publicado en formato digital: Prof. Aníbal Marcelo Mignone y Prof. Alejandra H. Torre Geraldi. SISTEMA URBANO ARGENTINO. Producción en Docencia. Revista Geográfica Digital. IGUNNE. Facultad de Humanidades. UNNE. Año 11. № 22. Julio - Diciembre 2014. Resistencia, Chaco. En: http://hum.unne.edu.ar/revistas/geoweb/default.htm 


\section{Los subsistemas}

- Noroeste: lineal

- Nordeste: Anular-lineal

- Central: radiocéntrico

- Cuyano: lineal

- Pampeano: radiocéntrico regular

- Patagónico: disperso

Fuente: Roccatagliata y Beguiristain, 1992; Erbiti, 1997. 


\section{Bibliografía}

- Banco Mundial. 1999. Informe sobre el Desarrollo Mundial 1998/99. Banco Mundial, Washington DC, 1999.

- Bortagaray, Lucía. 1992. "Las etapas de la ocupación del territorio argentino". Roccatagliata (coord.). La Argentina. Geografía general y los marcos regionales. 2da.ed., Planeta, Buenos Aires. 147-168.

- Erbiti, Cecilia. 1993. "El sistema espacial. Estructura de los asentamientos". Roccatagliata (coord.). Geografía económica argentina. El Ateneo, Buenos Aires. 44-69.

- Erbiti, Cecilia. 1997. "El sistema de asentamientos". Roccatagliata (coord.). Geografía económica Argentina. Temas. $2^{\circ}$ edición. El Ateneo. Buenos Aires. 96- 120.

- Erbiti, Cecilia. 2008. "Un sistema urbano en transformación. Metapolización, metropolización y ciudades intermedias; dinámicas". Roccatagliata (coord.). Argentina. Una visión actual y prospectiva desde la dimensión territorial. Emecé Editores S.A., Buenos Aires.217-254.

- Foschiatti, Ana María. 2003. La población del nordeste argentino. Universidad Nacional del Nordeste, Resistencia.

- INDEC. 2013. Censo Nacional de Población, Hogares y Viviendas 2010. Disponible en Internet: www.indec.gov.ar. Visitado el 20 de febrero de 2014

- Lindenboim, Javier y Damián Kennedy. 2003. "Continuidad y cambios en la dinámica urbana de Argentina". En: VII Jornadas Argentinas de Estudios de Población (AEPA). Universidad Nacional de Tucumán, Tafí del Valle. Tucumán. Tomo II: 1001- 1017.

- Meichtry, Norma C. 1994. "Sociedad y Alta Primacía en el Sistema Urbano Argentino." En: Anuario de Estudios Urbanos № 1.

- Meichtry, Norma. 1998. "Urbanización, alta primacía y desarrollo. Consideraciones conceptuales". En: Temas de población. Cuaderno de docentes $\mathbf{N}^{\circ}$ 7. IIGHI-CONICET, Resistencia.

- ONU (Organización de las Naciones Unidas). 1978. Factores determinantes y consecuencias de las tendencias demográficas. Nueva York, Volumen 1.

- Roccatagliata, Juan y Susana Beguiristain. 1992. Urbanización y sistema urbano. Roccatagliata (coord.). La Argentina. Geografía general y los marcos regionales. 2da.ed.. Planeta, Buenos Aires. 332-351.

- Usach, Natalia y Rubén Garrido. 2009. "Los cambios en el sistema urbano argentino: especialización y diversidad". En: Congreso de la Asociación Española de Ciencia Regional. XXXV Reunión de Estudios Regionales. 26 y 27 de noviembre de 2009. Disponible en Internet: www.reunionesdeestudiosregionales.org/valencia2009/htdocs/pdf/p168.pdf. Visitado el 20 de diciembre de 2013.

- Vapñarsky, Cesar A. y Néstor Gorojovsky. 1990. El Crecimiento Urbano en la Argentina. Buenos Aires, Grupo Editor Latinoamericano.

- Velázquez, Guillermo. 2000. Calidad de vida en la Argentina. Centro de Investigaciones Geográficas, Universidad Nacional del Centro de la Prov. de Buenos Aires. Tandil.

- Zarate Martín, Antonio. 1990. El sistema urbano de los países del Río de la Plata. En: Espacio, Tiempo y Forma. Serie VI. Geografía, T. 3 : 195-214. El espacio de los contenidos digitales de la UNED, España. Disponible en Internet: http://espacio.uned.es/fezleserv. php?pid=bibliuned:ETFSerie6-88B4D36E-AA8C-8445-C33B-05357B2E00CA\&dsID=Documento.pdf. Visitado el 12 de marzo de 2013.

Publicado en formato digital: Prof. Aníbal Marcelo Mignone y Prof. Alejandra H. Torre Geraldi. SISTEMA URBANO ARGENTINO. Producción en Docencia. Revista Geográfica Digital. IGUNNE. Facultad de Humanidades. UNNE. Año 11. No 22. Julio - Diciembre 2014. Resistencia, Chaco. En: http://hum.unne.edu.ar/revistas/geoweb/default.htm 